\title{
Vacancies in GaN bulk and nanowires: Effect of self-interaction corrections
}

\author{
Damien J. Carter ${ }^{1,2}$, Martin Fuchs ${ }^{3}$ and Catherine Stampfl ${ }^{4}$ \\ E-mail: stampfl@physics.usyd.edu.au \\ ${ }^{1}$ Nanochemistry Research Institute, Curtin University, PO Box U1987, Perth, WA \\ 6845, Australia \\ 2 iVEC, 21 Dick Perry Avenue, Kensington, WA 6151, Australia \\ 3 Fritz-Haber-Institut der Max-Planck-Gesellschaft, Faradayweg 4-6, D-14195 \\ Berlin-Dahlem, Germany \\ ${ }^{4}$ School of Physics, The University of Sydney, Sydney, NSW 2006, Australia
}

\begin{abstract}
We investigate gallium and nitrogen vacancies in gallium nitride (GaN) bulk and nanowires using self-interaction corrected pseudopotentials (SIC). In particular, we examine the band structures to compare and contrast differences between the SIC results and standard density functional theory (DFT) results using a generalised gradient approximation (GGA) [Phys. Rev. Lett. 77, 3845 (1996)] functional. For pure nanowires, we observed similar trends in the band gap behaviour, with the gap decreasing for increasing nanowire diameters (with larger band gaps using SIC pseudopotentials). For gallium vacancies in bulk GaN and GaN nanowires, SIC results are similar to DFT-GGA results, albeit with larger band gaps. Nitrogen vacancies in bulk GaN show similar defect-induced states near the conduction band, whilst a lower lying defect state is observed below the valence band for the DFT-GGA calculations and above the valence band for the SIC results. For nitrogen vacancies in GaN nanowires, similar defects state are observed near the conduction band, however, while the SIC calculations also show a defect state/s above the valence band, we were unable to locate this state for the DFT-GGA calculations (possibly because it is hybridised with edge states and buried below the valence band).
\end{abstract}

PACS numbers: 73.22.-f, 73.61.Ey, 71.55.-i 
In recent years there has been a surge of interest in semiconductor nanowires due to the unique optoelectronic, thermal, chemical and mechanical properties of these materials.[1] There is a vast array of potential applications of single crystalline nanowires with exciting new research in areas such as solar cells, [2] nanoelectronic batteries, [3] and nanowire lasers.[4] Despite significant advances, a number of challenges still remain to be overcome before nanowires can achieve widespread use, including issues of impurities in the synthesis of nanowires, how to synthesize large numbers of nanowires in arrangements required by computer circuits and how to integrate nanowires with current semiconductor-based electronics. Gallium nitride ( $\mathrm{GaN})$ is a widely studied III-V semiconductor in micro- and optoelectronic devices and GaN nanowires have been synthesised for a number of potential optoelectronic applications including ultravioletblue light-emitting diodes, nanowire lasers and spintronic devices.[4, 5, 6]

Native point defects control many aspects of a semiconductors behaviour, so a detailed understanding of their behaviour is crucial. Native point defects in bulk GaN have been examined in a number of theoretical studies. $[7,8,9,10,11,12,13,14]$ In bulk GaN, vacancies are the most prevalent native point defect, with nitrogen vacancies typically behaving as shallow donors, and gallium vacancies as acceptors.[12] The nitrogen vacancy is reported to be the dominant defect in GaN across the whole range of Fermi-level positions in the band gap,[14] while gallium vacancies have been found to induce a local magnetic moment.[15] Recent electrical transport measurements of GaN nanowires suggest that the Fermi level is pinned at the surface, leading to model of surface conductivity based on electron-hole recombination.[16]

To date there have been limited theoretical investigations of defects in GaN nanowires, $[17,18]$ and few theoretical studies of the properties of pure GaN nanowires. $[19,20,21,22,23,24]$ Results using functionals based on the generalised gradient approximation (GGA) are believed to be more reliable than those using the local density approximation (LDA), which is known to give too high/low formation energies. In a previous DFT-GGA investigation of vacancies in GaN nanowires[17] we reported, based on formation energies, that nitrogen and gallium vacancies prefer to form at the edges of nanowires, and that (in the neutral charge state) multiple vacancies prefer to cluster together than remaining well separated. This finding is supported by a recent DFT-LDA study by Wang et al.[18] who found that the most stable location for single vacancies, interstitials and anti-sites in GaN wires is also at, or near, the edge of nanowires.

In the present study we address the electronic structure, i.e. defect levels and band structure, of vacancies in bulk GaN and GaN nanowires, using self-interaction corrected pseudopotentials (SIC) pseudopotentials. It is well-known that conventional DFT-LDA and DFT-GGA run into certain limitations in describing the electronic structure of bulk semiconductors as well as (strongly localised) defects, when the Kohn Sham levels are interpreted as one-electron excitation energies (as measured e.g. in photoemission). Spurious self-interaction and the lack of derivative discontinuity in the exchange-correlation potential are notorious in this context. $G W$ quasi-particle calculations have been shown to overcome these difficulties, but their application to 
systems with localised valence states such as $\mathrm{GaN}$ is still debated,[25, 26, 27] and, in particular for defect systems, is computationally too demanding. Self-interaction corrections have been shown to cure some of these deficiencies[28, 29, 30] and improve the conventional DFT description without increasing the computational workload. An alternative approach to solving the self-interaction problem is the hybrid functional of Heyd, Scuseria and Ernzerhof (HSE), [31, 32] which has been applied successfully to study systems such as $\mathrm{AgO},[33] \mathrm{Cu}_{2} \mathrm{O},[34]$ transparent conducting oxychalcogenides,[35] and Mn doping in both GaN[36] and Ge.[37] To date, the HSE functional does not appear to have been used to study vacancies in the bulk or nanostructures of GaN. Here we apply the SIC to the calculation of the electronic structure of Ga- and N-vacancy defects in GaN nanowires and compare them to bulk GaN.

Atomic geometries of bulk GaN and nanowires are calculated with the DFT-GGA functional of Perdew, Burke and Ernzerhof (PBE) et al.[38] using the SIESTA[39] code. Orbitals are described using a double-zeta basis set with polarisation functions, confined using an energy shift of $0.01 \mathrm{Ry}$, and the $3 d$ electrons for gallium are explicitly included in the valence configuration. Hartree and exchange-correlation energies are evaluated using a grid cutoff of 300 Ry. The SIESTA method does not include symmetry, so all vacancy solutions in this study allowed for symmetry breaking. The electronic structure is then obtained from single-point band structure calculations, using DFTGGA and SIC pseudopotentials with the ABINIT[40] code, employing a planewave basis set with a kinetic energy cutoff of $50 \mathrm{Ry}$. The SIC band structure is calculated using SIC pseudopotentials and the DFT-LDA functional of Perdew and Wang et al..[41] Self-interaction corrections are included for the Ga $3 d$ and $\mathrm{N} 2 s$ and $2 p$ states using the pseudopotential scheme proposed by Vogel et al.[28, 42] Specifically, we use the Troullier-Martins[43] scheme as implemented in the fhipp code[44] to generate the SIC pseudopotentials:

$$
V_{l}^{\mathrm{PP}-\mathrm{SIC}}(r)=V_{l}^{\mathrm{PP}-\mathrm{LDA}}(r)+\delta V_{l}^{\mathrm{SIC}}(r),
$$

where $V_{l}^{\mathrm{PP}-\mathrm{LDA}}$ are standard DFT-LDA pseudopotentials and

$$
\begin{aligned}
& \delta V_{l}^{\mathrm{SIC}}(r)=-\theta\left(r_{\text {cut }}-r\right) \times \\
& \left(V^{\text {Hartree }}\left(\left[n_{l}\right], r\right)+V^{\mathrm{xc}-\mathrm{LDA}}\left(\left[n_{l}\right], r\right)-\frac{1}{r_{\text {cut }}}\right)
\end{aligned}
$$

are the short-range SIC potentials obtained from a self-consistent solution of the pseudoatom with the (spherically averaged) orbital densities $n_{l}$. The SIC pseudopotentials are transformed into and employed in their fully separable KleinmanBylander form.[28, 42, 45, 46]

For wurtzite bulk GaN calculations we sample the Brillouin zone with a $6 \times 6 \times 6 \mathrm{k}$ point mesh. The optimised wurtzite GaN lattice constants are $a=3.28 \AA, c=5.31 \AA$ and $u=0.378$, comparing well to experimental values of $a=3.19 \AA, c=5.19 \AA$ and $u=0.377$.[47] The band gap is $1.44 \mathrm{eV}$. A detailed discussion of the bulk properties of GaN, $a$-Ga and $\mathrm{N}_{2}$, including bulk modulus, cohesive energies, heat of formation and band structure has been previously reported.[21] The SIC band gap is calculated to be $4.17 \mathrm{eV}$. This 
compares well with the band gaps of $4.0 \mathrm{eV}[42]$ and $4.26 \mathrm{eV}[48]$ reported in other SIC studies. The band gap has also been reported as $3.52 \mathrm{eV}[49]$ using the exact-exchange method and as values ranging from 3.13-3.80 eV [50, 51, 52, 53, 54, 55] using the manybody perturbation theory in the $G W$ approximation. The experimental band gap is $3.50 \mathrm{eV} \cdot[56]$

We previously examined the atomic and electronic structure of pure GaN nanowires in the [0001] growth direction using DFT-GGA calculations. [21] These nanowires ranged in diameter from $9.5 \AA$ (48 atoms) to $35.0 \AA$ (432 atoms), as illustrated in Fig. 1A. Using the relaxed geometries from the DFT-GGA calculations of the 4 smallest diameter wires $(9.5$ to $21.0 \AA$ ), we calculate the SIC band structures. The Brillouin zone of these calculations is sampled using a $1 \times 1 \times 3 \mathrm{k}$-point mesh. In the plane normal to the nanowire growth direction, we use vacuum regions of approximately $20 \AA$ to ensure periodic images do not interact. For pure nanowires we saturate the dangling bonds at the surfaces of the nanowires with fictitious fractional-charge atoms $(0.75 e$ to saturate nitrogen atoms, and $1.25 \mathrm{e}$ to saturate gallium atoms).

The relative band gap as a function of nanowire diameter is illustrated in Fig. 1B, for both DFT-GGA and SIC calculations. For both SIC and DFT-GGA approaches, there is a decrease in band gap with increasing diameter, eventually approaching the respective bulk band gap values. As expected, Fig. 1B also illustrates that larger band gaps are obtained using the SIC method compared to DFT-GGA. In Fig. 1C we plot the relationship between the band gap and $1 / d^{2}$, where $d$ is the nanowire diameter. When the nanowire diameter is larger than approximately $19 \AA$, the band gaps follow closely the $1 / d^{2}$ proportionality relationship for the band gap change, which is characteristic of the finite-depth square-well model.[57] The close match between the trends for the SIC and the DFT-GGA band gaps of the nanowires illustrates that while standard DFT technique systematically underestimate band gaps, the qualitative trend is the same.

To study single vacancies in bulk GaN, we used a 96-atom supercell and fully optimised the atomic geometry. The resulting minimum energy structures are used for single point calculations of the SIC band structures. For the single and multiple gallium and nitrogen vacancies in [0001] GaN nanowires with SIC, we use $15.9 \AA$ diameter (216 atoms) nanowires, with fully relaxed geometries.[17] We consider single, double and triple nitrogen vacancies, and single and double gallium vacancies. The Brillouin zone in these calculations is sampled using a $1 \times 1 \times 3 \mathbf{~ k}$-point mesh. For investigations of vacancies in nanowires, the dangling bonds at the wire surfaces are not saturated, similar to other DFT investigations examining defects/doping in nanowires, including GaN, $[17,18,58,59] \mathrm{ZnO}[60]$ and $\mathrm{TiO}_{2} \cdot[61]$

In GaN nanowires we find the most stable vacancy configurations, for both single and multiple vacancies, are located at the edges of the nanowires. Furthermore, for the case of multiple vacancies, we find the most stable configurations are where the vacancies are in close proximity. In Figure 2 we show the atomic geometries of the most stable gallium and nitrogen two-vacancy configurations. The most stable nitrogen two-vacancy configuration (Fig. 2A) produces a separation distance of $2.65 \AA$ between two gallium 


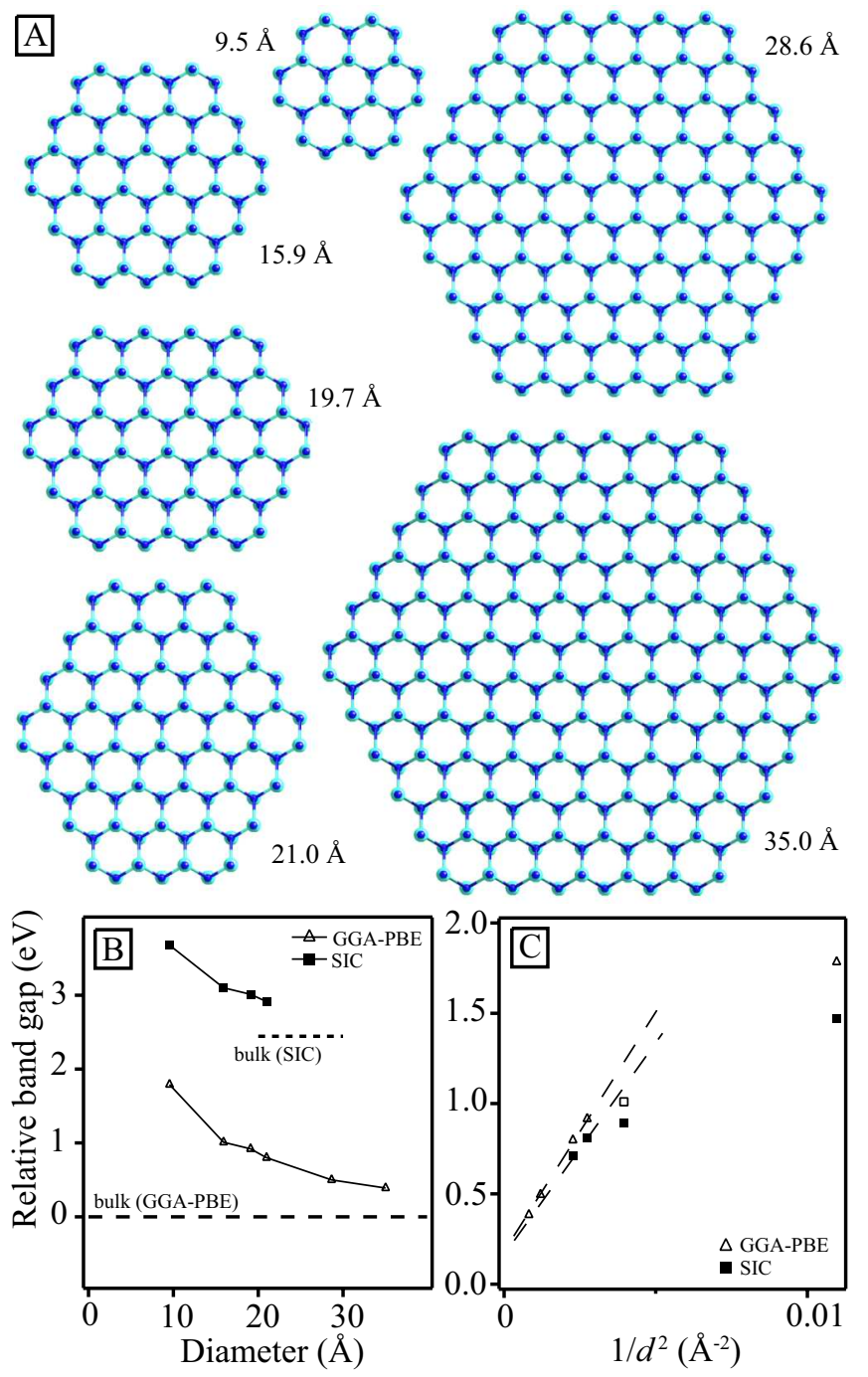

Figure 1. (A) Saturated nanowires in the [0001] direction that have hexagonal shaped cross-sections, viewed along the wire direction. The diameter of each size nanowire is labelled, with nitrogen and gallium atoms represented by the dark (blue) and light (aqua) spheres, respectively. (B) Relative bands gap as a function of nanowire diameter as obtained using SIC and DFT-GGA calculations. For comparison, the position of the SIC bulk band gap is also illustrated by the small, horizontal, dashed line. (C) Relative band gaps as a function of $1 / d^{2}$, where $d$ is the nanowire diameter. The dashed lines indicate the linear regions in each case. The SIC band gaps are relative to the bulk DFT-LDA band gap.

atoms near the vacancy locations, noticeably shorter than the 3.28 Adistance between gallium atoms in bulk GaN. The most stable gallium two-vacancy configuration (Fig. 2B) shows that three nitrogen atoms near the vacancies form an $\mathrm{N}_{3}$ trimer-like configuration (similar to an azide), with nitrogen-nitrogen separation distances of 1.30 and $1.41 \AA$. Further detail can be found in Ref. [17].

Figures 3 and 4 show schematic representations from DFT-GGA calculations[17] of the defect levels for nitrogen and gallium vacancies, respectively. For both nitrogen 


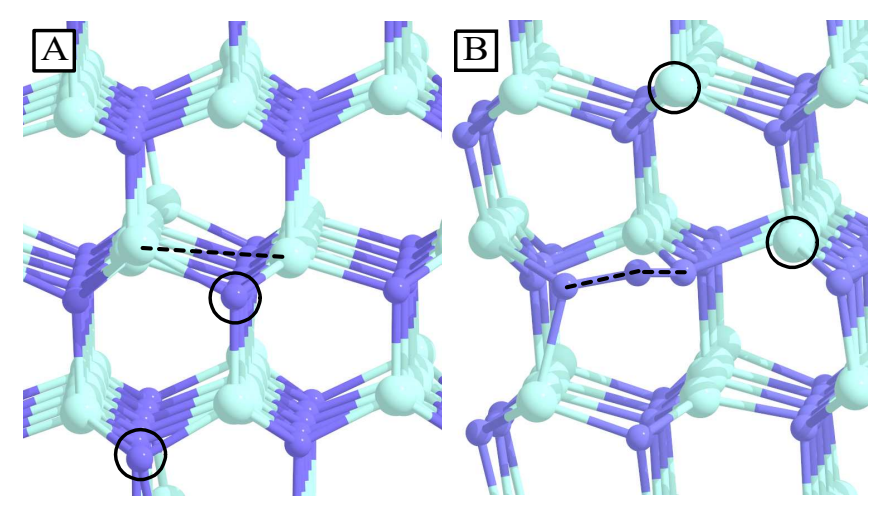

Figure 2. The most stable (a) nitrogen two-vacancy and (b) gallium two-vacancy configurations in the 216-atom nanowire. Nitrogen and gallium atoms are indicated by the small blue (dark) and the large aqua-coloured (light) spheres, respectively. The dashed line in (a) indicates a gallium-gallium separation distance of $2.65 \AA$, while the two dashed lines in (b) indicate nitrogen-nitrogen distances of 1.30 and $1.41 \AA$. The open circles represent the location of the vacancy atoms.

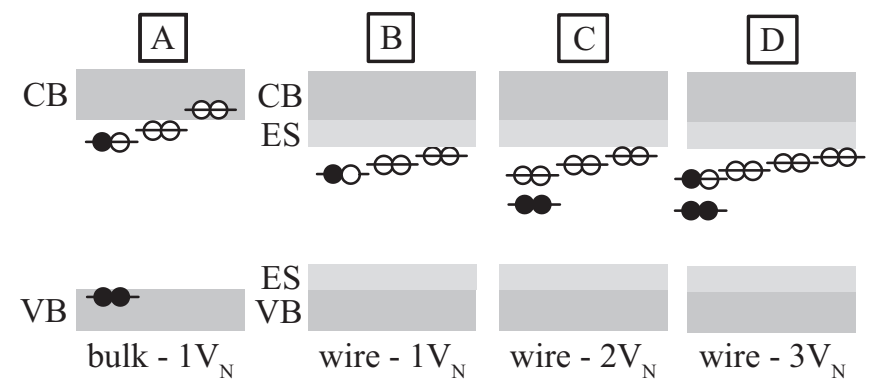

Figure 3. Schematic representation of the defect induced levels for (A) a single nitrogen vacancy in a 96-atom bulk GaN, and for nitrogen (B) one- (C) two- and (D) three-vacancy configurations in a 216-atom nanowire. Filled and open circles denote electrons and holes, respectively. "CB", "VB" and "ES" represent the conduction band, valence band and edge states, respectively.

and gallium vacancies in the nanowires, we observed that the defect states are near the edge state (ES) regions, rather than close to the valence band maximum (VBM) or conduction band minimum (CBM) as in bulk GaN. We attributed this to the presence of dangling bond states at the edges of nanowires.[17] Comparing nitrogen vacancies in bulk GaN to the nanowire, see Fig. 3, a notable difference is that bulk GaN has a localised defect state near the valence band (VB) that was not observed for the vacancies in the nanowire. We observe similar behaviour for gallium vacancies in bulk GaN and nanowires, see Fig. 4, with defect states close to the VBM in the bulk, and close to the ES region near the VBM in nanowires. In order to determine which states in the band structure belonged to the defect, the bulk, or edge states, we calculated the spatial distribution of the wavefunctions at the $\Gamma$-point.

We now investigate the SIC band structures for nitrogen and gallium vacancies in bulk GaN and GaN nanowires. The DFT-GGA and SIC band structures for a single 
$\mathrm{A}$

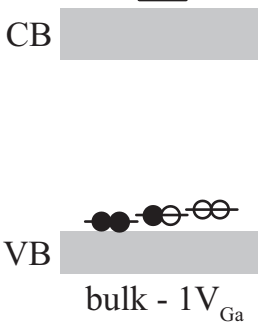

$\mathrm{B}$
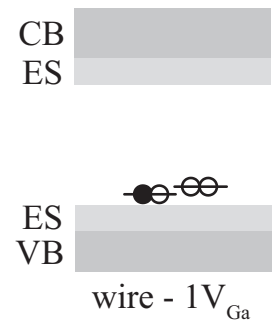

C

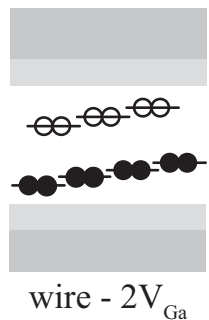

Figure 4. Schematic representation of the defect induced levels for (A) a single gallium vacancy in a 96-atom bulk GaN, and for gallium (B) one- and (C) two- vacancy configurations in a 216-atom nanowire. Filled and open circles denote electrons and holes, respectively. "CB", "VB" and "ES" represent the conduction band, valence band and edge states, respectively.

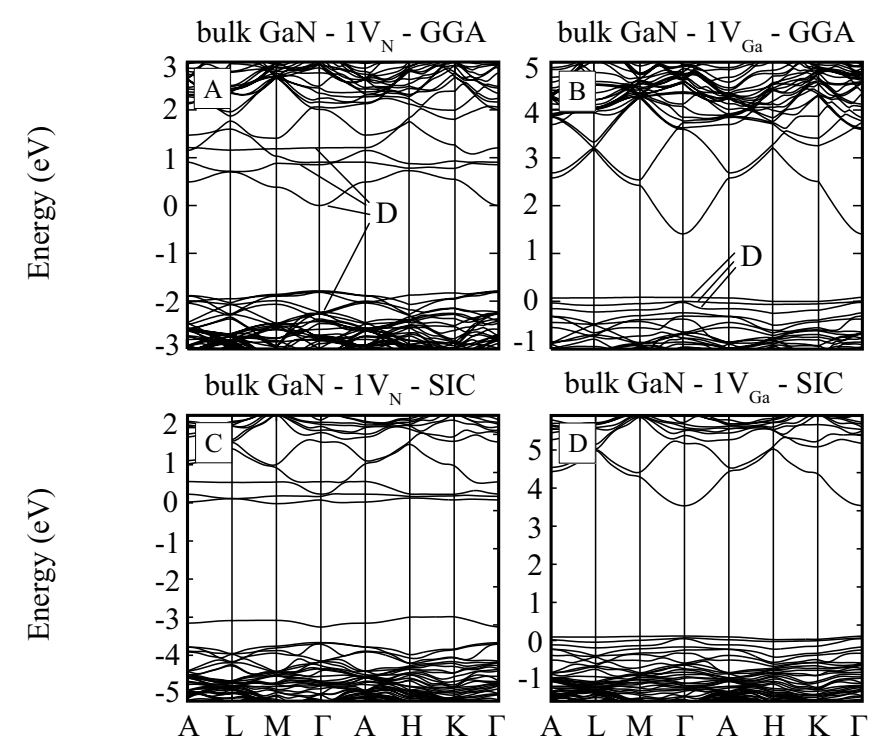

Figure 5. DFT-GGA band structure of the 96-atom wurtzite GaN supercell containing a single (A) nitrogen and (B) gallium vacancy, in the neutral charge state. SIC band structure of the 96-atom wurtzite GaN supercell containing a single (C) nitrogen and (D) gallium vacancy, in the neutral charge state. The energy zero is set at the highest occupied state and defect states are labelled with a D for Figs. A and B.

nitrogen vacancy in bulk GaN are shown in Figs. 5A and 5C, respectively. Four defectinduced singlet states are found in DFT-GGA calculations (Fig. 5A), with three near the CBM and one singlet state fully occupied below the VBM. The SIC band structure (Fig. 5C) is qualitatively similar (albeit with a larger band gap) with defect states in the band gap in the region of the CBM, however the lower defect state is not buried below the VBM, but rather is located just above it. Previous DFT studies of GaN have suggested that the conduction and valence band edges are predominantly of Ga $4 \mathrm{~s}$ and N $2 p$ character, respectively. [48, 21] The SIC pseudopotentials have a small affect 


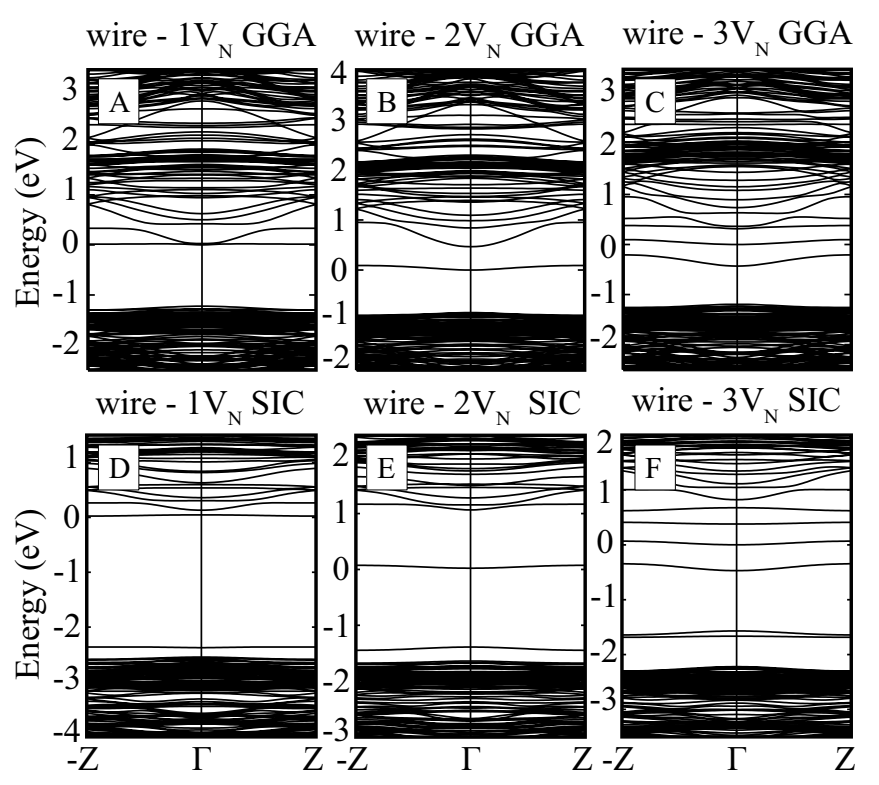

Figure 6. DFT-GGA band structures of the 216-atom GaN [0001] nanowire containing (A) single, (B) double, and (C) triple nitrogen vacancies. SIC band structures of the 216-atom GaN [0001] nanowire containing (A) single, (B) double, and (C) triple nitrogen vacancies. The energy zero is set at the highest occupied state.

on the location of the CB edges, but have a much larger effect on the VB edges as reported elsewhere.[48] Using the DFT-LDA, the Ga $3 d$ bands are located in the region of the $\mathrm{N} 2 s$ bands, leading to spurious $s$ - $d$ hybridisation, and reduced band gaps. The use of SIC corrects the $3 d$ bands to approximately $3 \mathrm{eV}$ lower energy than the $\mathrm{N} 2 s$ bands, decreasing the $s$ - $d$ hybridisation and the closed shell repulsion is reduced leading to larger band gaps.[48] With both GGA and LDA calculations of vacancies, the $s$ - $d$ hybridisation leads to reduced band gaps with position of the VB closer to the CB than it should be. As a result the defect state for the nitrogen vacancy that is in the region of the VBM becomes buried below the VBM. With SIC significantly increasing the band gap, the VB is further from the $\mathrm{CB}$, revealing the defect state just above the VBM.

The DFT-GGA and SIC band structures for a single gallium vacancy in bulk GaN are shown in Figs. 5B and 5D, respectively. Three defect-induced singlet states were found in DFT-GGA calculations (Fig. 5B), with all states located in the band gap region, just above the VBM. The SIC band structure (Fig. 5D) appears qualitatively similar to the DFT-GGA results with defect states near the VBM, but with an enlarged band gap.

We now examine SIC band structures for single and multiple vacancies in a $15.9 \AA$ diameter (216 atom) GaN [0001] nanowire. The DFT-GGA band structures for single, double and triple nitrogen vacancies are shown in Figs. 6A, 6B and 6C, respectively. The corresponding SIC band structures are shown in Figs. 6D, 6E and 6F, respectively. For the upper lying defect-induced states in the band gap, the SIC and DFT-GGA band structures are qualitatively similar (albeit with a larger band gap for the former), 

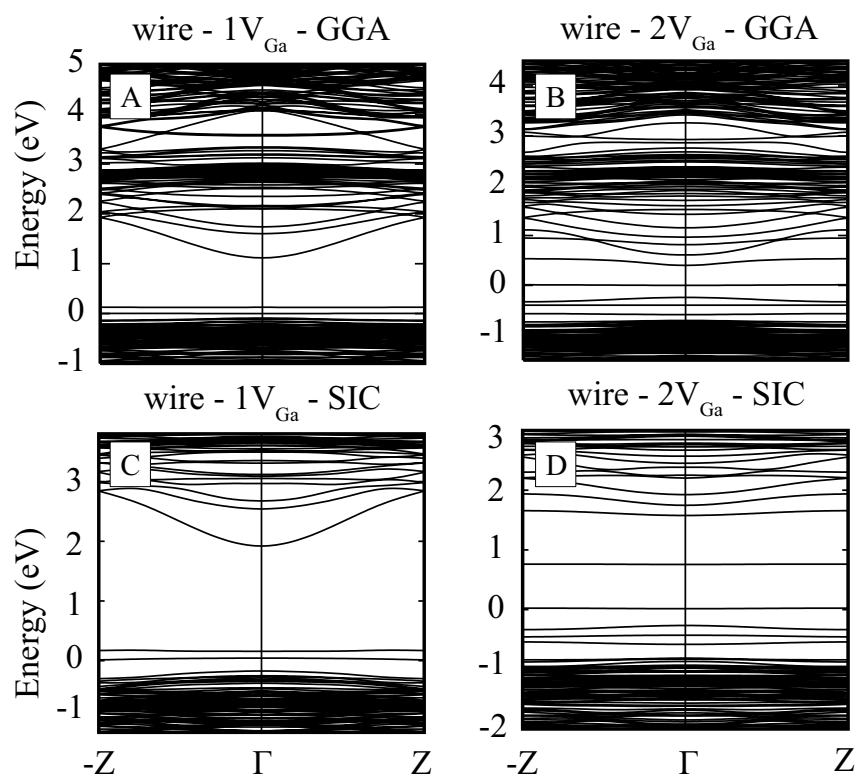

Figure 7. DFT-GGA band structures of the 216-atom GaN [0001] nanowire containing (A) single, and (B) double gallium vacancies. SIC band structures of the 216-atom GaN [0001] nanowire containing (C) single, and (D) double gallium vacancies. The energy zero is set at the highest occupied state.

however as observed with bulk GaN, the SIC band structure also shows a single defect state just above the VBM. Interestingly, as the number of nitrogen vacancies increases from one to two, the defect state close to the VBM moves higher into the band gap. Increasing to three vacancies, the defect states move further into the band gap and a second defect-induced state is also observed in close proximity to the first one. We note that earlier DFT-GGA investigations[17] could not clearly identify a defect state in the ES region near the VBM. Whilst several states did display some localised character, the closeness of bands near the VBM, combined with the edge states from dangling bonds at the edges of nanowires, made identification of a purely local vacancy defect state below the VBM very difficult. As found with a nitrogen vacancy in bulk GaN, SIC helps correct spurious $s$ - $d$ hybridisation which causes the VB to be closer to the CB than it actually should be. With SIC, the VB and edge states are lower in energy compared to the defect state from the nitrogen vacancy, revealing the previously buried, and unidentified defect states.

The DFT-GGA band structures for single and double gallium vacancies are shown in Figs. 7A and 7B, respectively. The corresponding SIC band structures are shown in Figs. 7C and 7D,respectively. The SIC and DFT-GGA results are qualitatively similar, with the SIC band gap larger, as expected.

In summary, we have investigated gallium and nitrogen vacancies in bulk GaN and GaN nanowires using self-interaction corrected pseudopotentials. Our results firstly show significantly improved agreement of the calculated band-gaps with experiment, for bulk GaN as well as for the nanowires for a wide range of diameters, compared 
to standard DFT calculations. For gallium vacancies in both the bulk and nanowires SIC results are similar to DFT-GGA results, albeit with a larger band gap, that is much closer to experimental values. In SIC calculations the nitrogen vacancy in bulk GaN produces a defect state just above the VBM in contrast to DFT-GGA calculations where this defect state is buried below the VBM. SIC calculations help correct spurious $s$ - $d$ hybridisation which occurs in DFT-GGA (and DFT-LDA) calculations and causes the VB to be closer to the CB than it actually should be, burying the defect level below the VBM. For single and multiple nitrogen vacancies in GaN nanowires, SIC and DFT-GGA calculations show similar upper lying defect-induced states in the band gap, however SIC calculations also show a defect state/s located just above the VBM, which was not found in DFT-GGA calculations. DFT-GGA calculations [17] were not able to identify this state near the VBM due to the VB region being complicated by edge states combined with the effects of the spurious $s$ - $d$ hybridisation (which reduces the separation between the $\mathrm{VB}$ and $\mathrm{CB}$ ).

SIC calculations find that the energy position of the nitrogen vacancy in the neutral state is notably higher than that obtained by standard DFT calculations. This result is significant because the energy position of a defect, as well as its occupancy and charge state, directly affects the predicted conductivity levels (e.g. the background n-type conductivity). Experimental studies of the electron mobility as a function of nanowire size (and which involve knowledge/estimation of the charge state of an assumed defect/dopant, as well as its energy position, e.g. nitrogen vacancies and/or oxygen impurities)[62] have been reported, and could be compared to, however this would involve calculation of formation energies, and charged defects which are outside the scope of the present manuscript. In a related context, a current controversy regarding the stability of the allowed charge states of the N-vacancy exists with some authors reporting the $3+$ charge state is stable[63] while others arguing it is not.[12] Our results suggest that this difference may not be just due to a difference in supercell size (as reported by Ref. [12]), but instead an inaccurate description of this defect state by standard DFT, which places the level too low where it is sensitive to, and erroneously influenced by, states at the valence band maximum.

The results presented in this paper illustrate that while DFT-GGA and SIC band structure for most defect levels of the $\mathrm{N}$ - and Ga-vacancies in both bulk GaN and GaN nanowires are qualitatively similar, inherent limitations in the standard DFT description cause a qualitative difference for the low lying localised nitrogen vacancy states. $G W$ calculations may be valuable for a more comprehensive investigation or more predictive power (e.g. charged defects), and are possible when combined with conventional DFT.[64, 65]

\section{Acknowledgments}

We gratefully acknowledge the National Computational Infrastructure (NCI) and the Australian Centre for Advanced Computing and Communications for the use of their 
facilities. This work was supported by the Australian Research Council.

\section{References}

[1] P. J. Pauzauskie and P. Yang. Nanowire photonics. Materials Today, 9:36-45, 2006.

[2] B. Tian, X. Zheng, T. J. Kempa, Y. Fang, N. Yu, G. Yu, J. Huang, and C. M. Lieber. Coaxial silicon nanowires as solar cells and nanoelectronic power sources. Nature, 449:885-889, 2007.

[3] C. K. Chan, H. Peng, G. Liu, K. Mcllwrath, X. F. Zhang, R. A. Huggins, and Y. Cui. Highperformance lithium battery anodes using silicon nanowires. Nature Nanotechnology, 3:31-35, 2008.

[4] J. C. Johnson, H.-J. Choi, K. P. Knutsen, R. D. Schaller, P. Yang, and R. J. Saykally. Single gallium nitride nanowire lasers. Nature Materials, 1:106-110, 2002.

[5] J.-R. Kim, H. M. So, J. W. Park, J.-J. Kim, J. Kim, C. J. Lee, and S. C. Lyu. Electrical transport properties of individual gallium nitride nanowires synthesized by chemical-vapor-deposition. Appl. Phys. Lett., 80:3548-3550, 2002.

[6] H.-J. Choi, H.-K. Seong, J. Chang, K.-I. Lee, Y.-J. Park, J.-J. Kim, S.-K. Lee, R. He, T. Kuykendall, and P. Yang. Single-crystalline diluted magnetic semiconductor gan:mn nanowires. Advanced Materials, 17:1351-1356, 2005.

[7] J. Neugebauer and C. G. Van de Walle. Atomic geometry and electronic-structure of native defects in gan. Phys. Rev. B, 50(11):8067-8070, 1994.

[8] P. Boguslawski, E. L. Briggs, and J. Bernholc. Native defects in gallium nitride. Phys. Rev. B, 51:R17255-R17258, 1995.

[9] C. H. Park and D. J. Chadi. Stability of deep donor and acceptor centers in gan, aln, and bn. Phys. Rev. B, 55:12995-13001, 1997.

[10] T. Mattila and R. M. Nieminen. Point-defect complexes and broadband luminescence in gan and aln. Phys. Rev. B, 55:9571-9576, 1997.

[11] I. Gorczyca, A. Svane, and N. E. Christensen. Theory of point defects in gan, aln, and bn: Relaxation and pressure effects. Phys. Rev. B, 60:8147-8157, 1999.

[12] C. G. Van de Walle and J. Neugebauer. First-principles calculations for defects and impurities: Applications to iii-nitrides. J. Appl. Phys., 95(8):3851-3879, 2004.

[13] A. Gulans, R. A. Evarestov, I. Tale, and C. C. Yang. Ab initio calculations of charged point defects in gan. Physica Status Solidi C, 2:507-510, 2005.

[14] M. G. Ganchenkova and R. M. Nieminen. Nitrogen vacancies as major point defects in gallium nitride. Phys. Rev. Lett., 96:196402, 2006.

[15] J. Hong. Local magnetic moment induced by ga vacancy defect in gan. J. Appl. Phys., 103:063907, 2008.

[16] R. Calarco, M. Marso, T. Richter, A. I. Aykanat, R. Meijers, A. V. D. Hart, T. Stoica, and H. Luth. Size-dependent photoconductivity in mbe-grown gan-nanowires. Nano Lett., 5:981-984, 2005.

[17] D. J. Carter and C. Stampfl. Atomic and electronic structure of single and multiple vacancies in gan nanowires from first-principles. Phys. Rev. B, 79:195302, 2009.

[18] Z. Wang, J. Li, F. Gao, and W. J. Weber. Defects in gallium nitride nanowires: First principles calculations. J. Appl. Phys., 108(4):044305, 2010.

[19] M.-H. Tsai, Z.-F. Jhang, J.-Y. Jiang, Y.-H. Tang, and L. W. Tu. Electrostatic and structural properties of gan nanorods/nanowires from first principles. Appl. Phys. Lett., 89:203101, 2006.

[20] A. Gulans and I. Tale. Ab initio calculation of wurtzite-type gan nanowires. Physica Status Solidi C, 4:1197-1200, 2007.

[21] D. J. Carter, J. D. Gale, B. Delley, and C. Stampfl. Geometry and diameter dependence of the electronic and physical properties of gan nanowires from first principles. Phys. Rev. B, $77: 115349,2008$.

[22] D. Q. Fang, A. L. Rosa, T. Frauenheim, and R. Q. Zhang. Band gap engineering of gan nanowires by surface functionalization. Appl. Phys. Lett., 94(7):073116, 2009. 
[23] P. Dev, H. Zeng, and P. Zhang. Defect-induced magnetism in nitride and oxide nanowires: Surface effects and quantum confinement. Phys. Rev. B, 82(16):165319, 2010.

[24] R. Agrawal and H. D. Espinosa. Giant piezoelectric size effects in zinc oxide and gallium nitride nanowires. a first principles investigation. Nano Lett., 11:786-790, 2011.

[25] P. Rinke, A. Qteish, J. Neugebauer, C. Freysoldt, and M. Scheffler. Combining gw calculations with exact-exchange density-functional theory: an analysis of valence-band photoemission for compound semiconductors. New J. Phys, 7:126, 2005.

[26] M. van Schilfgaarde, T. Kotani, and S. V. Faleev. Adequacy of approximations in gw theory. Phys. Rev. B, 74:245125, 2006.

[27] H. Jiang, R. I. Gomez-Abal, P. Rinke, and M. Scheffler. First-principles modeling of localized d states with the gw@lda+u approach. Phys. Rev. B, 82:045108, 2010.

[28] D. Vogel, P. Kruger, and J. Pollmann. Self-interaction and relaxation-corrected pseudopotentials for ii-vi semiconductors. Phys. Rev. B, 54:5495-5511, 1996.

[29] A. Droghetti, C. D. Pemmaraju, and S. Sanvito. Predicting d0 magnetism: Self-interaction correction scheme. Phys. Rev. B, 78:140404, 2008.

[30] S. Lany and A. Zunger. Polaronic hole localization and multiple hole binding of acceptors in oxide wide-gap semiconductors. Phys. Rev. B, 80:085202, 2009.

[31] J. Heyd, G. E. Scuseria, and M. Ernzerhof. J. Chem. Phys., 118:8207, 2003.

[32] J. Heyd, G. E. Scuseria, and M. Ernzerhof. J. Chem. Phys., 124:219906, 2006.

[33] J. P. Allen, D. O. Scanlon, and G. W. Watson. Phys. Rev. B, 81:161103, 2010.

[34] D. O. Scanlon, B. J. Morgan, G. W. Watson, and A. Walsh. Phys. Rev. Lett., 103:096405, 2009.

[35] D. Scanlon and G. Watson. Chem. Mater., 21:5435, 2009.

[36] A. Stroppa and G. Kresse. Unraveling the jahn-teller effect in mn-doped gan using the heydscuseria-ernzerhof hybrid functional. Phys. Rev. B, 79:201201, 2009.

[37] A. Stroppa, G. Kresse, and A. Continenza. Phys. Rev. B, 83:085201, 2011.

[38] J. P. Perdew, K. Burke, and M. Ernzerhof. Generalized gradient approximation made simple. Phys. Rev. Lett., 77:3865-3868, 1996.

[39] J. M. Soler, E. Artacho, J. D. Gale, A. Garcia, J. Junquera, P. Ordejon, and D. Sanchez-Portal. The siesta method for ab initio order-n materials simulation. Journal of Physics: Condensed Matter, 14:2745-2779, 2002.

[40] X. Gonze, J.-M. Beuken, R. Caracas, F. Detraux, M. Fuchs, G.-M. Rignanese, L. Sindic, M. Verstraete, G. Zerah, F. Jollet, M. Torrent, A. Roy, M. Mikami, P. Ghosez, J.-Y. Raty, and D. C. Allan. First principles computation of material properties: the abinit software project. Comp. Mater. Sci., 25:478-492, 2002.

[41] J. P. Perdew and Y. Wang. Accurate and simple analytic representation of the electron-gas correlation energy. Phys. Rev. B, 45:13244-13249, 1992.

[42] D. Vogel, P. Kruger, and J. Pollmann. Structural and electronic properties of group-iii nitrides. Phys. Rev. B, 55:12836-12839, 1997.

[43] N. Troullier and J. L. Martins. Efficient pseudopotentials for plane-wave calculations. Phys. Rev. B, 43:1993-2006, 1991.

[44] M. Fuchs and M. Scheffler. Ab initio pseudopotentials for electronic structure calculations of polyatomic systems using density-functional theory. Computer Physics Communications, 119:67-68, 1999.

[45] L. Kleinman and D. M. Bylander. Efficacious form for model pseudopotentials. Phys. Rev. Lett., 48:1425-1428, 1982.

[46] Our pseudoization radii for the $\mathrm{Ga}\left(3 d^{10}, 4 s^{2}, 4 p^{1}\right)$ and $\mathrm{N}\left(2 s^{2}, 2 p^{3}, 3 d^{0}\right)$ states are $(1.5,1.5,1.5)$ and $(2.2,2.4,2.1)$ bohr respectively. SIC potentials are included for the Ga $3 d$ and the $\mathrm{N} 2 s$ and $2 p$ states with a (smooth) cut off at $r_{\text {cut }}=7.3$ bohr. The Ga $4 s$ and the N $3 d$ potentials are used as the local parts in the Kleinman-Bylander potentials which we checked to be free of ghost states [28].

[47] H. Schulz and K. H. Thiemann. Crystal structure refinement of aln and gan. Solid State Commun., 
23(11):815-819, 1977.

[48] A. Filippetti and N. A. Spaldin. Self-interaction-corrected pseudopotential scheme for magnetic and strongly-correlated systems. Phys. Rev. B, 67:125109, 2003.

[49] A. Qteish, A. I. Al-Sharif, M. Fuchs, M. Scheffler, S. Boeck, and J. Neugebauer. Role of semicore states in the electronic structure of group-iii nitrides: An exact-exchange study. Phys. Rev. B, $72: 155317,2005$.

[50] A. Rubio, J. L. Corkill, M. L. Cohen, E. L. Shirley, and S. G. Louie. Quasiparticle band structure of aln and gan. Phys. Rev. B, 48:11810-11816, 1993.

[51] M. Oshikiri and F. Aryasetiawan. Quasiparticle energy calculations on ii(zn)-vi(o, s, se) and iii(al,ga)-v(n) semiconductors in the wurtzite structure. J. Phys. Soc. Jpn., 69:2113-2120, 2000.

[52] T. Kotani and M. van Schilfgaarde. All-electron gw approximation with the mixed basis expansioned on the full-potential lmto method. Solid State Commun., 121:461-465, 2002.

[53] D. Cociorva, W. G. Aulbur, and J. W. Wilkins. Quasiparticle calculations of band offsets at alnâe"gan interfaces. Solid State Commun., 124:63-66, 2002.

[54] P. Rinke, M. Scheffler, A. Qteish, M. Winkelnkemper, D. Bimberg, and J. Neugebauer. Band gap and band parameters of inn and gan from quasiparticle energy calculations based on exactexchange density-functional theory. Appl. Phys. Lett., 89:161919, 2006.

[55] A. Svane, N. E. Christensen, I. Gorczyca, M. van Schilfgaarde, A. N. Chantis, and T. Kotani. Quasiparticle self-consistent gw theory of iii-v nitride semiconductors: Bands, gap bowing, and effective masses. Phys. Rev. B, 82:115102, 2010.

[56] B. Monemar. Fundamental energy gap of gan from photoluminescence excitation spectra. Phys. Rev. B, 10:676-681, 1974.

[57] A. D. Yoffe. Low-dimensional systems: quantum size effects and electronic properties of semiconductor microcrystallites (zero-dimensional systems) and some quasi-two-dimensional systems. Advances in Physics, 42:173-262, 1993.

[58] Q. Wang, Q. Sun, and P. Jena. Ferromagnetism in mn-doped gan nanowires. Phys. Rev. Lett., 95:167202, 2005.

[59] Q. Wang, Q. Sun, P. Jena, and Y. Kawazoe. Ferromagnetic gan-cr nanowires. Nano Lett., 5:1587$1590,2005$.

[60] D. Q. Fang and R. Q. Zhang. Size effects on formation energies and electronic structures of oxygen and zinc vacancies in zno nanowires: A first-principles study. J. Appl. Phys., 109(4):044306, 2011.

[61] J. Nisar, C. M. Araujo, and R. Ahuja. Water interaction with native defects on rutile tio2 nanowire: Ab initio calculations. Appl. Phys. Lett., 98(=):083115, 2011.

[62] A. Motayed, A. V. Davydov, S. N. Mohammad, and J. Melngailis. J. Appl. Phys., 104:024302, 2008.

[63] K. Laaksonen, M. G. Ganchenkova, and R. M. Nieminen. J. Phys.:Condens. Matter, 21:015803, 2009.

[64] P. Rinke, A. Janotti, M. Scheffler, and C. G. Van de Walle. Defect formation energies without the band-gap problem: Combining density-functional theory and the gw approach for the silicon self-interstitial. Phys. Rev. Lett., 102:026402, 2009.

[65] C. Van de Walle and A. Janotti. Advances in electronic structure methods for defects and impurities in solids. Phys. Status Solidi B, 248:19-27, 2011. 